\title{
Crecimiento y mortalidad del pez Canthidermis sufflamen (Perciformes: Balistidae) de los archipiélagos Los Frailes y Los Testigos, Venezuela
}

\author{
Mayra Alarcón ${ }^{1}$, Nora Eslava ${ }^{2}$, Leo Walter González ${ }^{2}$ \& Francisco Guevara ${ }^{2}$ \\ 1. Universidad de Oriente. Escuela de Ciencias Aplicadas del Mar. Departamento de Acuacultura. Boca del Río, isla de \\ Margarita, Venezuela; jennifer.m.alarcon.r@gmail.com \\ 2. Universidad de Oriente. Instituto de Investigaciones Científicas. Área de Biología y Recursos Pesqueros. Boca del \\ Río, Isla de Margarita, Venezuela; neslava20@yahoo.es, 1wgc25@gmail.com, franciscogm2002e@hotmail.com
}

\author{
Recibido 14-IX-2016. Corregido 06-III-2017. Aceptado 05-IV-2017.
}

\begin{abstract}
Growth and mortality of the triggerfish Canthidermis sufflamen (Perciformes: Balistidae) from Los Frailes and Los Testigos Archipelagos, Venezuela. Canthidermis sufflamen has become a low cost food resource in high demand in El Tirano fishing community, and captures have been increasing in recent years. Since there is a lack of information on this resource population dynamics in Venezuela, the goal of this research was to provide some biological-fishery parameters, and has as general objective to determine growth and mortality of the triggerfish of Los Frailes and Los Testigos Archipelagos, Federal Territories of Venezuela. For this, a total of 3595 specimens were collected from El Tirano commercial fishing, every week, from May 2012 to April 2013. We determined total length (cm), total weight $(\mathrm{g})$, and sex for each specimen. No sexual dimorphism was shown with respect to length $\left(\mathrm{t}_{\mathrm{s}}=-0.96 ; \mathrm{p}>0.05\right)$, so one length-weight ratio was established for both sexes: $\mathrm{W}=0.164 * \mathrm{~L}^{2.26}$; and they both exhibited minor allometric growth. Growth was estimated by analysis of the frequency distribution of lengths, using FiSAT software. Growth parameters, estimated by the von Bertalanffy model, were considered slow, being $\mathrm{L}_{\infty}=61.69 \mathrm{~cm}, \mathrm{~W}_{\infty}=1868.402 \mathrm{~g}, \mathrm{k}=0.36$ / year, and $\mathrm{t}_{\mathrm{o}}=-0.654$ years. The maximum age was $\mathrm{A}_{0.95}=8$ years. The growth index $\left(\varnothing^{\prime}\right)$, showed a value of 3.14 . The exploitation rate $\mathrm{E}=0.72$ / year, using total mortality values $\mathrm{Z}=2.67$ / year, natural $\mathrm{M}=0.73$ / year and from fishing $\mathrm{F}=1.93$ / year, indicates that $C$. sufflamen is being over-exploited by the artisanal fishers of El Tirano. We recommend following some harvest guidelines that will reduce mortality rate due to fishing. Rev. Biol. Trop. 65 (3): 858-867. Epub 2017 September 01.
\end{abstract}

Key words: Canthidermis sufflamen, growth, mortality, Los Frailes and Los Testigos Archipelagos, Venezuela.

En la dinámica de poblaciones, la comprensión y caracterización del crecimiento individual es de suma importancia porque este proceso, junto con la supervivencia, determina la magnitud del recurso. Del mismo modo, al obtener un conocimiento detallado sobre el patrón de crecimiento de una especie se logrará estimaciones más precisas sobre el tamaño de la población y su disponibilidad para la explotación (Arreguín, Sánchez, \& Defeo, 1991; Castillo, Eslava, \& González, 2011). Otro aspecto fundamental es la mortalidad que está relacionada con las dos principales causas a las que están expuestos los peces a lo largo de su vida: la mortalidad natural cuya acción es causada por depredación, enfermedad y vejez, y la mortalidad por pesca originada, por la acción del hombre (Csirke, 1980).

La familia Balistidae comprende unas 40 especies en todo el mundo, 10 de ellas se conocen en aguas colombianas, resaltando Canthidermis maculata y Melichthys niger por estar presentes en el Caribe y el Pacífico (Acero, Mejía, \& Santos-Acevedo, 2002). También en las islas occidentales del archipiélago canario, España, algunas de estas especies tropicales 
son de importancia comercial, principalmente Canthidermis sufflamen y Decapterus macarellus (Brito, 2008). Por otro lado, en la isla de Trinidad situada $1160 \mathrm{~km}$ de la costa brasileña se han reportado mortalidades masivas hasta de $99 \%$, posiblemente, vinculadas a la proliferación de algas tóxicas, cambios bruscos en la temperatura, ingreso de masas de agua dulce de los ríos o flujos causados por el exceso de lluvia a zonas costeras arrecifales característicos de esta zona (Pinheiro, Gasparini, \& Joyeux, 2010).

En el estado Nueva Esparta, Venezuela, $C$. sufflamen es una especie relativamente abundante y de bajo precio, por lo que tiene una alta demanda en el mercado local (González, 2015, com. pers.). El tamaño de la flota artesanal de El Tirano durante la temporada de pesca 2012, estuvo constituida por 83 embarcaciones con motor fuera de borda llamados "peñeros" de 6 a $9 \mathrm{~m}$ de eslora, con las cuales se obtuvo una captura total de $786110 \mathrm{~kg}$. C. sufflamen ocupó el tercer lugar de la captura total con nasa, arte de pesca tipo trampa con un total de 84638 kg (González, Eslava, Guevara, Díaz
\& Rodríguez, 2017, en prensa). Sin embargo, no se conocen estudios sobre crecimiento y mortalidad, información de índole periódica indispensable en la evaluación y toma de decisiones en la administración pesquera. Por tal razón, se decidió realizar esta investigación, con el fin de contribuir al conocimiento de la dinámica poblacional de este recurso para su aprovechamiento de manera sustentable.

\section{MATERIALES Y MÉTODOS}

Los ejemplares de $C$. sufflamen fueron capturados con nasa, arte de pesca tipo trampa utilizada por la comunidad de pescadores de El Tirano que faenan en los archipiélagos Los Frailes y Los Testigos. Los Frailes está ubicado al noreste de la isla de Margarita del estado

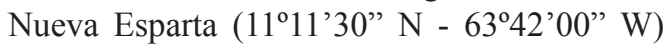
(Fig. 1). Los Testigos, ubicado en el sector nororiental del Mar Caribe venezolano a una latitud $11^{\circ} 20^{\prime} 47^{\prime \prime}$ - $11^{\circ} 24^{\prime} 46^{\prime \prime} \mathrm{N} \& 63^{\circ} 02^{\prime} 34^{\prime \prime}$ - 630' $21^{\prime \prime} \mathrm{W}$ formando parte de las Dependencias Federales de Venezuela (Villa, 1967; Cervigón, 1995). Por su situación geográfica,

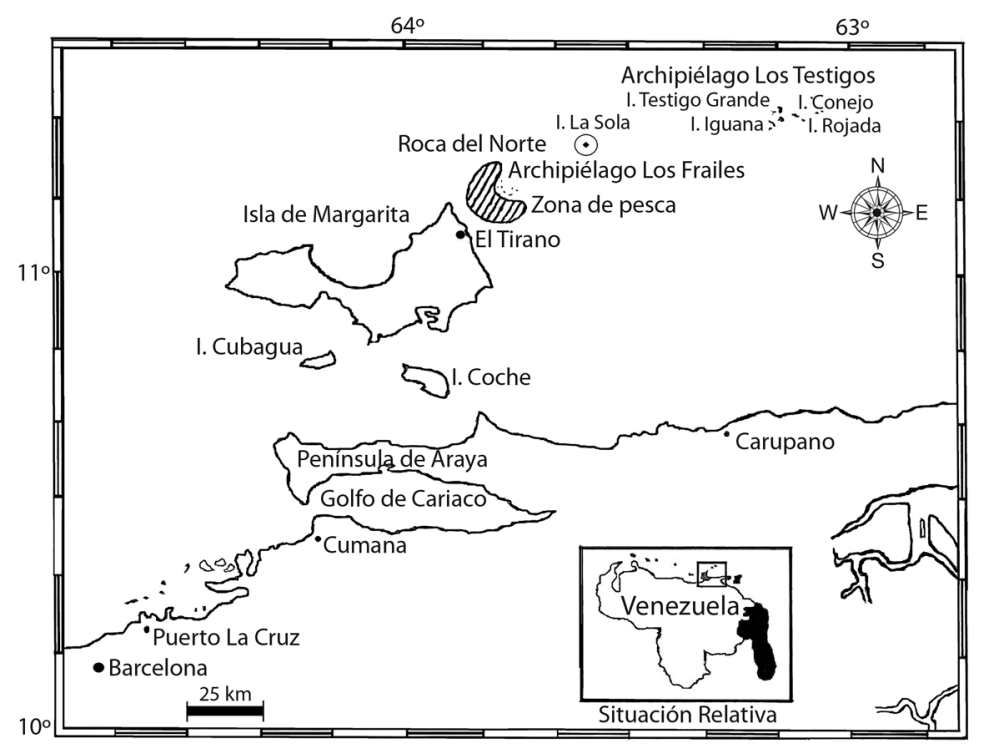

Fig. 1. Zona de pesca de los Archipiélagos Los Frailes y Los Testigos. Venezuela. Modificado de González, Eslava y Guevara (2001).

Fig. 1. The Fishing zone from Los Frailes and Los Testigos Archipelagos, Venezuela. Modification of González, Eslava and Guevara (2001). 
ambos archipiélagos juegan un papel preponderante en los planes estratégicos de Venezuela, que genera al país una Zona Económica Exclusiva rica en recursos pesqueros, turísticos y ecológicos (Ciro \& Zambrano, 2007).

Los muestreos se realizaron semanalmente en la playa de El Tirano, de mayo 2012 a abril de 2013, obteniéndose un mínimo de 200 y un máximo de 383 individuos por mes, incluyendo los meses de lluvia que son de mayo a septiembre. A cada ejemplar se le tomó la longitud total (L) en centímetros, el peso total (P) en gramos con una balanza marca Royal Universal con capacidad de $5 \mathrm{~kg}$ y apreciación de 1 g y la identificación del sexo se realizó mediante la observación macroscópica de las gónadas (Billings \& Munro, 1974). Para determinar diferencias significativas entre la longitud media por sexo, se realizó la prueba de t-student (Zar, 2009). Se estableció la relación longitud-peso mediante la ecuación propuesta por Ricker (1975): $\mathrm{P}=$ $\mathrm{a}^{*} \mathrm{~L}^{\mathrm{b}}$ donde $\mathrm{P}$ es el peso total sin eviscerar en $\mathrm{g}$, L es la longitud total en cm, "a" es la intersección con el eje de las ordenadas y "b" es la pendiente de la curva. Se probó la hipótesis del crecimiento isométrico mediante una prueba $\mathrm{t}_{\mathrm{s}}$ (Walpole, Myers, Myers, \& Ye, 2007) con un nivel de significancia de $\alpha=0.05$; teniendo como hipótesis $\mathrm{H}_{\mathrm{o}}: \mathrm{b}=3$ y $\mathrm{H}_{\mathrm{a}} \mathrm{b} \neq 3$, los resultados de esta prueba fueron usados para determinar el tipo crecimiento: isométrico $(b=$ $3)$, alométrico minorante $(\mathrm{b}<3)$ o alométrico mayorante $(b>3)$.

La estimación del crecimiento se basó en las distribuciones mensuales de frecuencia de longitudes con un rango de $2 \mathrm{~cm}$, para ello se usó el software FiSAT (Gayanilo, Sparre, \& Pauly, 1996). En primer lugar, se determinó una longitud asintótica $\left(\mathrm{L}_{\infty}\right)$ preliminar aplicando el método de Powell (1979) y Wetherall (1986), que permitió estimar el coeficiente de crecimiento $(\mathrm{k})$ a través de la rutina ELEFAN I, la misma que se ajustó a la función de von Bertalanffy (1938) por un método no paramétrico, a través de interacciones, donde la curva óptima que cruza el mayor número de picos posibles es seleccionada por una bondad de ajuste que se determina por el coeficiente de la suma de las modas disponibles entre la suma de modas explicadas (de León, 2005). Para la optimización de los parámetros de $\mathrm{L}_{\infty} \mathrm{y}$ de $\mathrm{k}$, se empleó el procedimiento de Gulland y Holt (1959) asociado en la misma rutina, la cual se realizó previo análisis de la progresión modal utilizando la descomposición de frecuencia de longitudes según el método de Bhattacharya (1967). Luego, se calculó $t_{0}$ de acuerdo a la formula anotada por Pauly (1979): $\log _{10}\left(-\mathrm{t}_{\mathrm{o}}\right)=$ $0.3922-0.2752 * \log _{10} \mathrm{~L}_{\infty}-1.038 * \log _{10} \mathrm{k}$, donde $\mathrm{t}_{\mathrm{o}}$ es la edad hipotética a la cual la longitud es cero, $\mathrm{L}_{\infty}$ es la longitud asintótica promedio máxima y $\mathrm{k}$, es la constante de crecimiento o coeficiente de curvatura. Para determinar la edad límite o longevidad de un pez, se utilizó la ecuación de Taylor (1958): $\mathrm{A}_{0.95}=\mathrm{t}_{\mathrm{o}}+$ $2.996 / \mathrm{k}$, donde $\mathrm{A}_{0.95}$ es la edad límite teórica o tiempo requerido para que el pez alcance el $95 \%$ de su longitud máxima $\left(\mathrm{L}_{\infty}\right)$. El peso asintótico de determinó mediante la ecuación anotada por Csirke (1980): $\mathrm{P}_{\infty}=\mathrm{a}^{*} \mathrm{~L}_{\infty}{ }^{\mathrm{b}}$ donde $\mathrm{P}_{\infty}$ es el peso asintótico o peso máximo. Una vez calculados los parámetros $\mathrm{L}_{\infty}, \mathrm{P}_{\infty}, \mathrm{k} \mathrm{y}_{\mathrm{o}}$, se procedió a establecer las curvas de crecimiento en longitud y peso, según el modelo de von Bertalanffy (1938): $\mathrm{L}=\mathrm{L}_{\infty}\left[1-\mathrm{e}^{\mathrm{k}(\mathrm{t}-\mathrm{to})}\right] ; \mathrm{P}=$ $\mathrm{P}_{\infty}\left[1-\mathrm{e}^{\mathrm{k}(\mathrm{t}-\mathrm{to})}\right]^{\mathrm{b}}$ porque se pueden incorporar fácilmente a modelos de evaluación de poblaciones (Gulland, 1971a). Se estableció el índice de crecimiento phi prima $\left(\varnothing^{\prime}\right)$ de acuerdo a la fórmula establecida por Pauly \& Munro (1984): $\varnothing^{\prime}=\log _{10} \mathrm{k}+2 \log _{10} \mathrm{~L}_{\infty}$.

La mortalidad total $\mathrm{Z}$ se calculó utilizando el método de la curva de captura linearizada (Pauly, 1983): $\operatorname{lnC}\left(\mathrm{L}_{1}, \mathrm{~L}_{2}\right) / \Delta \mathrm{t}\left(\mathrm{L}_{1}, \mathrm{~L}_{2}\right)$ $=\mathrm{c}-\mathrm{Z} * \mathrm{t}\left(\mathrm{L}_{1}+\mathrm{L}_{2} / 2\right)$. La mortalidad natural M se evaluó mediante la ecuación de Pauly (1980): $\log _{10}(\mathrm{M})=-0.0066-0.279 * \log _{10} \mathrm{~L}_{\infty}$ $+0.6543 * \log _{10} \mathrm{k}+0.4634 * \log _{10} \mathrm{~T}$, para una temperatura superficial media del agua de $\mathrm{T}$ $=27^{\circ} \mathrm{C}$ de los archipiélagos Los Frailes y Los Testigos, obtenida del Centro Internacional para la Investigación del Fenómeno de El Niño (CIIFEN). La mortalidad por pesca F se calculó aplicando la ecuación citada por Sparre y Venema (1997) $\mathrm{F}=\mathrm{Z}$ - M, y la tasa de explotación 
E según la ecuación propuesta por Gulland (1971b) $\mathrm{E}=\mathrm{F} / \mathrm{Z}$.

\section{RESULTADOS}

Se examinó un total de 3595 individuos con longitudes comprendidas entre $13 \mathrm{~cm}$ y $58 \mathrm{~cm}$ (Media $=30.58 \mathrm{~cm} \pm 4.54)$ y pesos que oscilaron entre 53 g y 2180 g (Media $=413.5$ $\mathrm{g} \pm 156.38$ ), observándose la menor y mayor longitud en el mes de enero 2013 (Cuadro 1). No se encontraron diferencias estadísticamente significativas para la longitud entre hembras y machos $\left(t_{s}=0.96 ; p>0.05\right)$, por lo que se trataron los sexos combinados, para la determinación de crecimiento y mortalidad. Las longitudes con mayor frecuencia estuvieron comprendidas entre los $29 \mathrm{~cm}$ y $33 \mathrm{~cm}$ (Fig. 2), siendo la de $30 \mathrm{~cm}$ la más abundante durante los meses de julio, agosto y noviembre (Fig. 3).
Crecimiento: La relación longitud-peso quedó establecida como $\mathrm{P}=0.167 * \mathrm{~L}^{2.26}$ siendo la intercepción $\mathrm{a}=0.167$ y la pendiente $\mathrm{b}=2.26$ significativamente diferente a $3\left(\mathrm{t}_{\mathrm{s}}=43.81 ; \mathrm{p}<\right.$ 0.05 ) (Fig. 4). La primera estimación de $\mathrm{L}_{\infty}=$ $61.69 \mathrm{~cm}$ fue utilizada como dato de entrada en la superficie de respuesta en el ELEFAN I para la estimación del coeficiente de curvatura, que luego de varias corridas se obtuvo un $\mathrm{k}=$ 0.36 / año. Posteriormente, se aplicó la rutina de (Gulland \& Holt, 1959) la cual proporcionó valores adicionales de $\mathrm{L}_{\infty}(49.15 \mathrm{~cm})$ y k $(2.329$ / año), que fueron descartados debido a que no correspondieron a las características biológicas de la especie. $E 1 \mathrm{t}_{\mathrm{o}}=-0.654$ años $\mathrm{y} \mathrm{k}=0.36$ / año calculados permitió determinar la edad máxima teórica $\left(\mathrm{A}_{0.95}\right)$ en 8 años. Con los valores de las constantes de la relación longitud-peso y $\mathrm{L}_{\infty}$ se estimó el $\mathrm{P}_{\infty}=1868.402 \mathrm{~g}$. La curva de crecimiento en longitud describió una curva de tipo

\section{CUADRO 1}

Ejemplares recolectados de Canthidermis sufflamen durante mayo 2012-abril 2013

en los archipiélagos Los Frailes y Los Testigos, Venezuela

TABLE 1

Canthidermis sufflamen specimens collected during May 2012-April 2013 from Los Frailes and Los Testigos Archipelagos, Venezuela

\begin{tabular}{lccccccc}
\multicolumn{1}{c}{ Mes/año } & $n$ & Min - Max & Media & DE \pm & Min - Max & Media & DE \pm \\
May-12 & 210 & $24-38$ & 30 & 2.83 & $196-700$ & 365 & 96.3 \\
Jun-12 & 313 & $16-45$ & 29 & 3.73 & $66-1187$ & 370 & 123.24 \\
Jul-12 & 370 & $19-49$ & 30 & 3.83 & $118-1228$ & 400 & 123.49 \\
Ago-12 & 383 & $14-45$ & 30 & 3.74 & $53-1168$ & 401 & 127.36 \\
Sep-12 & 323 & $16-45$ & 29 & 5.7 & $64-1000$ & 387 & 171.8 \\
Oct-12 & 282 & $21-51$ & 30 & 4.42 & $149-1624$ & 414 & 164.23 \\
Nov-12 & 382 & $18-55$ & 30 & 5.01 & $71-1369$ & 399 & 161.79 \\
Dic-12 & 315 & $19-44$ & 31 & 4.46 & $113-885$ & 447 & 174.88 \\
Ene-13 & 204 & $13-58$ & 30 & 5.7 & $56-2180$ & 411 & 226.79 \\
Feb-13 & 222 & $25-53$ & 36 & 5.45 & $219-1568$ & 547 & 214.26 \\
Mar-13 & 351 & $22-48$ & 31 & 4.07 & $168-946$ & 396 & 117.97 \\
Abr-13 & 240 & $18-45$ & 31 & 5.62 & $89-1118$ & 425 & 174.26 \\
TOTAL & 3595 & & 30.58 & 4.54 & & 413.5 & 156.36 \\
\hline
\end{tabular}

$n=$ número ejemplares examinados; Min = valor mínimo; Max = valor máximo;

Media $=$ valor medio $; \mathrm{DE}=$ desviación estándar.

$n=$ number of specimens examined; Min = minimum value; Max = maximum value;

Media $=$ mean value DE $=$ standard deviation . 


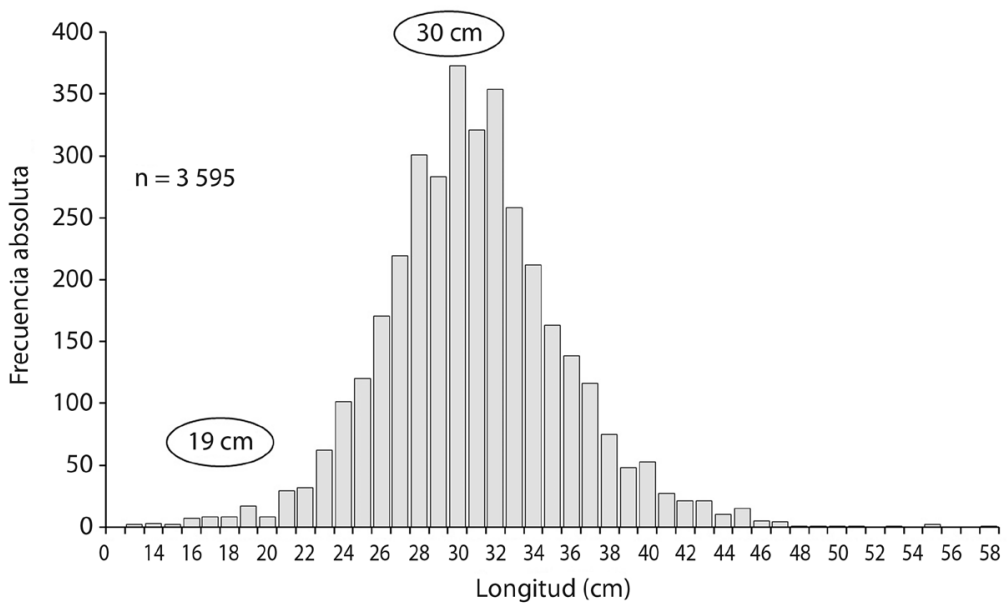

Fig. 2. Distribución de frecuencia de longitudes de Canthidermis sufflamen.

Fig. 2. Frequency distribution of lengths of Canthidermis sufflamen.
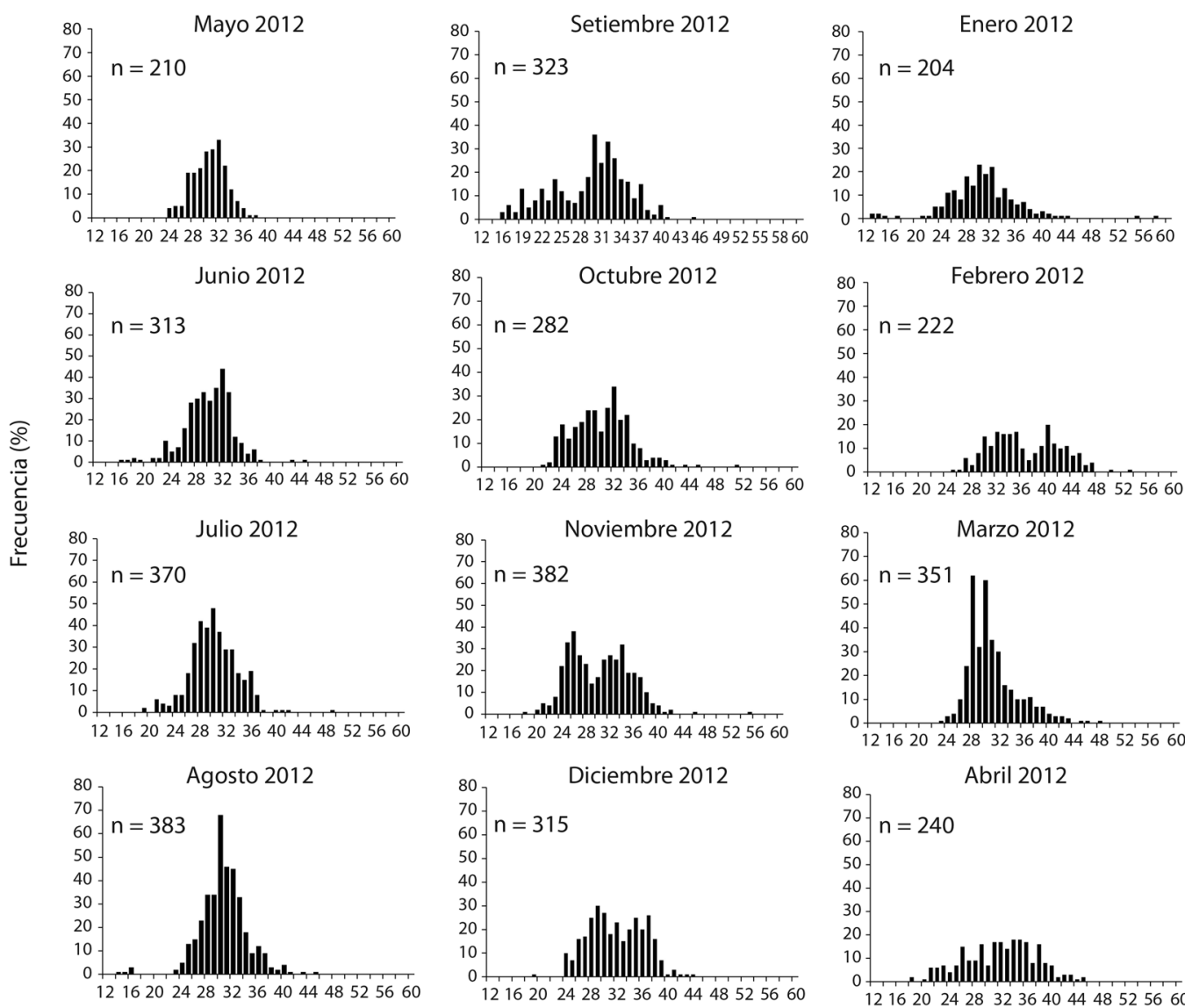

Febrero 2012
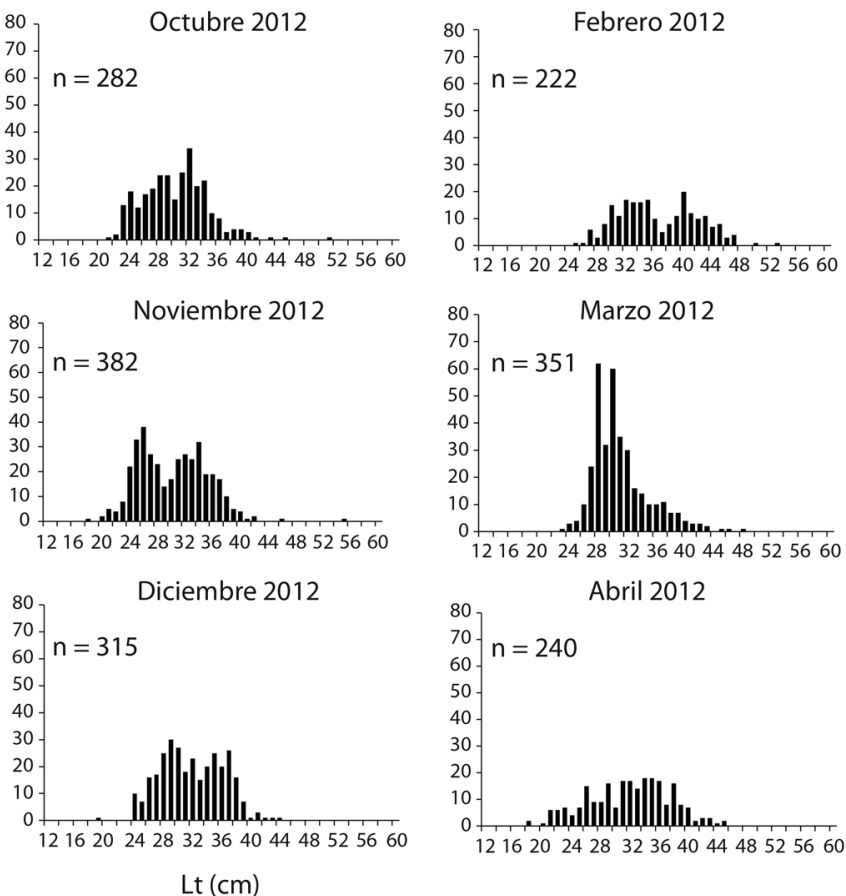

Fig. 3. Distribución de frecuencia de longitudes mensuales de Canthidermis sufflamen durante mayo 2012-abril 2013.

Fig. 3. Frequency distribution of monthly lengths of Canthidermis sufflamen during May 2012-April 2013. 


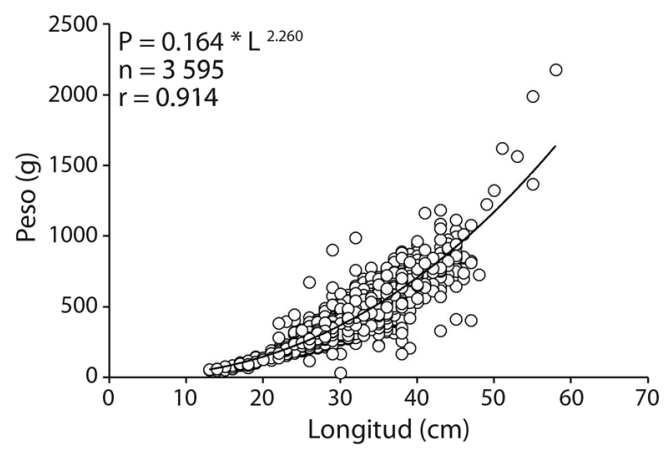

Fig. 4. Relación longitud-peso de Canthidermis sufflamen. Fig. 4. Length-weight relationship of Canthidermis sufflamen.

exponencial, acelerado en el primer año de vida, alcanzando aproximadamente, el $44 \%$ de su longitud asintótica, y luego disminuyó lentamente hasta lograr la longitud máxima teórica (Fig. 5A). Mientras que la curva de crecimiento en peso delineó una curva de tipo sigmoideo, donde el primer año de vida tuvo un crecimiento lento, para luego crecer de forma exponencial y así finalmente, conseguir su peso asintótico (Fig. 5B). El índice de crecimiento phi prima fue $\varnothing^{\prime}=3.14$.

Mortalidad: La mortalidad natural $\mathrm{M}$ establecida para Canthidermis sufflamen fue de 0.73 / año y la mortalidad total $Z$ de 2.67 / año (Fig. 6), con las cuales se pudo determinar la mortalidad por pesca $\mathrm{F}=1.93$ / año, y la tasa de explotación $\mathrm{E}=0.72$ / año.

\section{DISCUSIÓN}

La ausencia de ejemplares pequeños en los muestreos pudo deberse, a dos probables causas: a) la selectividad del arte de pesca utilizado (nasa) que controla la retención y fuga de los peces, debido a que la entrada o embudo regula el tamaño máximo y la malla de revestimiento limita la talla mínima retenida (Pope, Margetts, Hamley, \& Akyuz, 1983; Slack-Smith, 2001), y b) la disponibilidad del recurso en el área de pesca, porque los juveniles se encuentran
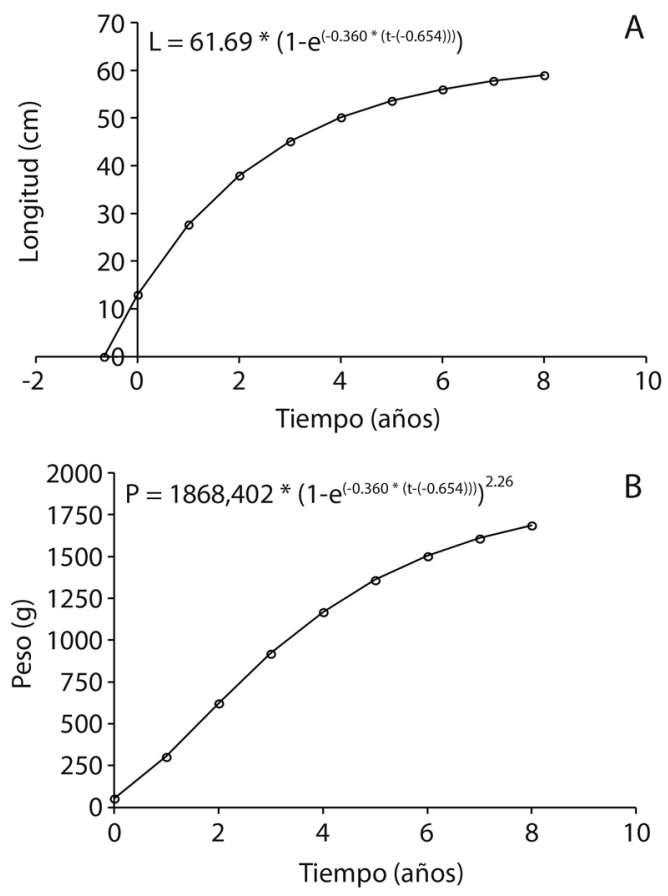

Fig. 5. (A) Curva de crecimiento en longitud de Canthidermis sufflamen según el modelo de von Bertalanffy (1938). (B) Curva de crecimiento en peso de Canthidermis sufflamen según el modelo de von Bertalanffy (1938).

Fig. 5. (A) Growth curve in length of Canthidermis sufflamen, according to the von Bertalanffy model (1938). (B) Growth curve in weight of Canthidermis sufflamen, according to the von Bertalanffy model (1938).

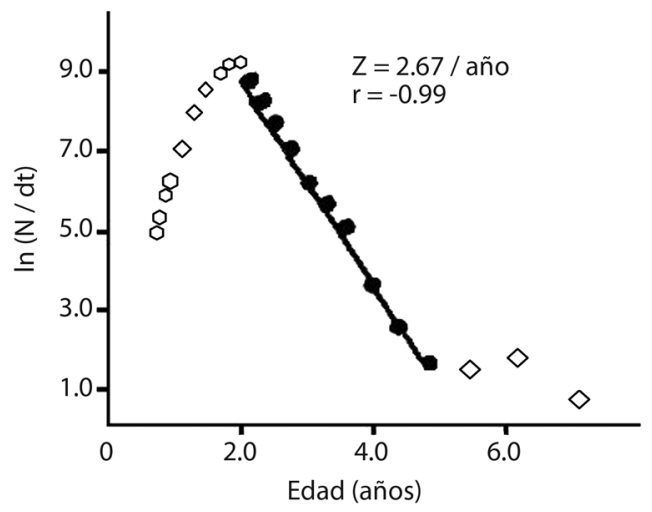

Fig. 6. Estimación de la mortalidad total (Z) de Canthidermis sufflamen a partir del método de la curva de captura linearizada.

Fig. 6. Estimation of total mortality $(Z)$ from a linearized catch curve method of Canthidermis sufflamen. 
en zonas de crecimiento hasta el desarrollo de las gónadas, momento en que se reclutan al stock de adultos, es decir, probablemente exista una segregación espacial en este organismo (Lindeman, 1989). Las mediciones máximas de longitud y peso examinadas en enero $(58 \mathrm{~cm}$ y $2180 \mathrm{~g}$ ) difieren a los reportados por (Lieske \& Myers, 1994) en el Atlántico (65 cm y 6100 g).

La relación longitud-peso evidenció un crecimiento alométrico minorante $(b<3)$ lo que significa que el pez es menos pesado para la longitud que tiene (Ehrhardt, 1981; Pauly, 1993; Zúñiga, 2009). Los valores de las constantes pueden variar entre las especies y estar relacionado con el tamaño de la muestra, época del año, alimentación, estado de madurez, factores ambientales y con el rápido crecimiento en las primeras etapas de vida, donde cada una de ellas puede presentar una determinada relación entre la longitud y el peso (Tresierra \& Culquichicón, 1993; Gómez, Guzmán, \& Chacón, 2002). Generalmente, las poblaciones de peces de menor tamaño utilizan la energía para crecer rápido con el objetivo de alcanzar pronto la madurez sexual y así evitar la extinción de la especie, debido a la alta explotación pesquera en una zona determinada (do Santos, Pessanha, da Costa, \& Araújo, 2004).

La falta de tallas pequeñas e individuos adultos podría ser la causa principal de subestimación del parámetro de $\mathrm{k}$ y sobreestimación de $\mathrm{L}_{\infty}$, debido a la correlación que existe entre los parámetros de crecimiento del modelo de von Bertalanffy (Cubillos, Aguayo, Neira, Sanhueza, \& Castillo-Jordán, 2009; Zúñiga, 2009). Las estimaciones de la longitud asintótica y el coeficiente de curvatura permitieron inferir que es un pez de crecimiento discretamente bajo, de acuerdo con los parámetros del ciclo de vida para poblaciones de peces reportados por Musick (1999). El valor negativo obtenido de $t_{0}$ indica que los individuos pequeños crecen más rápidamente (de manera exponencial) que los adultos. Una información de parámetros biológicos de larvas podrían ayudar a realizar una mejor estimación de $\mathrm{t}_{\mathrm{o}}$, parámetro asociado biológicamente con la eclosión del huevo, que técnicamente se refiere al origen de la curva o la edad teórica del organismo a la cual tiene una longitud igual a cero (Prince, Lee, \& Conser, 1987). Si no se tiene una buena representación del grupo de edad 0 , se puede sobre-estimar $\mathrm{L}_{\infty}$ y sub-estimar $\mathrm{k}$ y $\mathrm{t}_{\mathrm{o}}$ (Cota, 2008). Las variaciones de los parámetros de crecimiento pueden deberse a diferencias en el hábitat y sus características ambientales particulares, como la temperatura superficial del mar (Charnov \& Gillooly, 2004) y el fotoperiodo, las cuales pueden variar estacionalmente y estar correlacionadas con la presión de los depredadores, cambios en la disponibilidad, abundancia y calidad del alimento (ciclos de producción del mar), factores intrínsecos de la especie (Carpenter, 2002), así como los regímenes de explotación a los que están sometidos (Bravo, 2007). Con respecto a las curvas de crecimiento individual en longitud y peso, Kaufmann (1981) y Maroñas (2006) explicaron que al comienzo el crecimiento es rápido, luego decrece suavemente, repitiéndose las pautas año a año porque depende del resultado de dos fuerzas opuestas, anabolismo y catabolismo (Zavatteri, 2010) lo que concuerda con el comportamiento en las curvas de crecimiento de $C$. sufflamen. No obstante el crecimiento en peso se va acelerando luego, hasta desarrollar una velocidad máxima cuando el pez ha alcanzado un peso que es aproximadamente $1 / 3$ de su peso máximo (Ricker, 1975; Csirke, 1980), lo que para $C$. sufflamen fue $623 \mathrm{~g}$.

Las mortalidades estimadas fueron altas $(\mathrm{M}=0.73$ / año, $\mathrm{F}=1.93$ / año y $\mathrm{Z}=2.67$ / año). La mortalidad natural $\mathrm{M}$, posiblemente, esté relacionada con la cantidad de depredadores y competidores existentes cuya abundancia está influenciada por la pesca (Sparre \& Venema, 1997), y, por otro lado, la estrategia de crecimiento durante las primeras etapas de vida, que permite a los individuos modificar la mortalidad natural (Tordecilla-Petro, SánchezBanda, \& Olaya-Nieto, 2005). En segundo lugar, la mortalidad por pesca $\mathrm{F}$ exhorta explorar el efecto de la pesca sobre la población de peces, asignando un coeficiente de proporcionalidad para las muertes debido a la pesca (Sparre \& Venema, 1997), y de esta manera 
poder distinguir con claridad entre esta mortalidad y aquellas causadas por otros factores. Ello significa que cada vez que un pez sobreviva a la mortalidad por pesca, tendrá que sobrevivir también a la mortalidad natural y viceversa, para llegar con vida al final de cada período, así de este modo se establece una competencia entre la mortalidad por pesca y otras causas de mortalidad (Csirke, 1980). En el caso de la mortalidad total $\mathrm{Z}$ explica un fuerte impacto de la pesca, cuyo efecto directo puede causar cambios en la abundancia, en relación a un ecosistema en equilibrio, y también en la estructura de tallas, con una reducción en las tallas máximas (Sparre \& Venema, 1997). Según Gulland (1971b), la tasa de explotación $\mathrm{E}=0.5 /$ año es el valor óptimo de una pesquería, por lo que se infiere que $C$. sufflamen está siendo sobre explotada debido a la alta valoración estimada de la tasa de explotación $(E=0.72$ / año). Por consiguiente, se sugiere regular la pesquería, reduciendo la tasa de mortalidad por pesca, teniendo en cuenta que es la tercera especie importante en cuanto a sus volúmenes de captura de la flota artesanal de El Tirano, según lo reportado por González et al. (2017)

Se concluye que $C$. sufflamen no evidencia dimorfismo sexual con respecto a la longitud y presenta un crecimiento lento con una alta tasa de explotación, lo cual demuestra que la especie está sobre explotada por la pesca artesanal de El Tirano; por lo que se sugiere continuar con los estudios de la dinámica de poblaciones para evaluar la pesquería con fines de manejo.

\section{AGRADECIMIENTOS}

A Juan Miguel Rodríguez, Presidente del Centro de Acopio "Virgen del Carmen" y a José Gregorio, encargado del camión cava "Bonanza" y su equipo de trabajo, y pescadores de la zona por su colaboración incondicional en este año de trabajo, facilitando los ejemplares antes de su comercialización. Al Consejo de Investigación de la Universidad de Oriente por el financiamiento parcial del Proyecto de Grupo: "Evaluación de la pesquería artesanal de la comunidad de El Tirano, isla de Margarita" Código CI-6-030603-1587 / 2009. A Clark Casler por la lectura y sugerencias al manuscrito.

\section{RESUMEN}

En la comunidad pesquera de El Tirano, estado Nueva Esparta, Venezuela, la especie Canthidermis sufflamen se ha convertido en un recurso con una alta demanda para el consumo debido su bajo costo, por lo que su captura ha venido incrementándose en los últimos años. Debido a la escasa información existente sobre la dinámica poblacional de este recurso, y con la finalidad de aportar algunos parámetros biológico-pesqueros se planteó como objetivo general determinar el crecimiento y mortalidad de Canthidermis sufflamen en los archipiélagos Los Frailes y Los Testigos, Dependencias Federales de Venezuela, durante el período mayo 2012 y abril 2013, se obtuvo un total de 3595 ejemplares capturados con nasa por la flota artesanal de El Tirano. A cada ejemplar se le tomó datos de longitud total $(\mathrm{cm})$, peso total $(\mathrm{g})$ y se determinó el sexo. Se comprobó que no existe diferencia sexual con respecto a longitud $\left(\mathrm{t}_{\mathrm{s}}=-0.96 ; \mathrm{p}>0.05\right)$, la relación longitud-peso para ambos sexos fue: $\mathrm{P}=0.164 * \mathrm{~L}^{2.26}$, lo que evidencia un crecimiento alométrico minorante. La estimación del crecimiento se basó en el análisis de la distribución de frecuencia de longitudes, con uso del software FiSAT. Los parámetros de crecimiento estimados $\left(\mathrm{L}_{\infty}=61.69 \mathrm{~cm}, \mathrm{P}_{\infty}=\right.$ $1868.402 \mathrm{~g}, \mathrm{k}=0.36 /$ año $\mathrm{y} \mathrm{t}_{\mathrm{o}}=-0.654$ años) mostraron un crecimiento lento. La edad límite fue $\mathrm{A}_{0.95}=8$ años. El índice de crecimiento $\left(\varnothing^{\prime}\right)$ obtuvo un valor de 3.14. La tasa de explotación $E=0.72 /$ año calculada a través de la mortalidad total $\mathrm{Z}=2.67$ / año, natural $\mathrm{M}=0.73$ / año y por pesca $\mathrm{F}=1.93$ / año, indica que $C$. sufflamen está siendo sobre explotada por la pesca artesanal de El Tirano; por consiguiente se sugiere regular la pesquería, reduciendo la tasa de mortalidad por pesca.

Palabras clave: Canthidermis sufflamen, crecimiento, mortalidad, archipiélago Los Frailes y Los Testigos, Venezuela.

\section{REFERENCIAS}

Acero, A., Mejía, L. S., \& Santos-Acevedo, M. (2002). Balistes vetula. In L. S. Mejía \& A. Acero (Eds.), Libro rojo de peces marinos de Colombia (pp. 118120). Bogotá: INVEMAR.

Arreguín, F., Sánchez, J. A., \& Defeo, O. (1991). Análisis del crecimiento de la almeja amarilla (Mesodesma mactroides) de la costa uruguaya en base a datos de composición por longitudes. Frente Marítimo, 9, $75-81$.

Bhattacharya, C. (1967). A simple method of resolution of a distribution into Gaussian components. Biometrics, $23,115-123$. 
Billings, C., \& Munro, J. (1974). The biology, ecology and bionomics of Caribbean reef fishes: Pomadasydae (Grunts). Part 5. Research Report from the Zoology Department, University of the West Indies, 3, 1-128.

Bravo, E. (2007). Crecimiento, mortalidad natural y madurez sexual del cuji, Haemulon aurolineatum (Cuvier $y$ Valenciennes, 1830) (Teleostei: Haemulidae) del sureste de la isla de Margarita (Tesis Pregrado). Universidad de Oriente, Boca del Río, Venezuela.

Brito, A. (2008). Influencia del calentamiento global sobre la biodiversidad marina de las Islas Canarias. En J. Alfonso-Carrillo (Ed.), Naturaleza amenazada por los cambios en el clima (Actas de la III Semana Científica Telésforo Bravo) (pp. 141- 161). Islas Canarias: Instituto de Estudios Hispánicos de Canarias.

Carpenter, K. E. (Ed.). (2002). The living marine resources of the Western Central Atlantic. Bony fishes part 2 (Opistognathidae to Molidae), sea turtles and marine mammals. FAO Species Identification Guide for Fishery Purposes and American Society of Ichthyologists and Herpetologists. (5 ed., Vol. 3, pp. 13752127). Rome, Italy: FAO.

Castillo, J., Eslava, N., \& González, L. W. (2011). Crecimiento del cangrejo Callinectes danae (Decápoda: Portunidae) de la Isla de Margarita, Venezuela. Revista de Biología Tropical, 59(4), 1525-1535.

Cervigón, F. (1995). Las Dependencias Federales. Caracas, Venezuela: Academia Nacional de la Historia.

Ciro, S., \& Zambrano, S. (2007). Estudio sedimentológico de las geoformas litorales acumulativas recientes en el Archipiélago Los Testigos, Dependencias Federales. Sapiens, Revista Universitaria de Investigación, 1317-5815.

Cota, J. (2008). Edad y crecimiento del marlín azul, Makaira nigricans, desembarcado en cabo San Lucas, B.C.S. México (Tesis Maestría). Centro Interdisciplinario de Ciencias Marinas, Instituto Politécnico Nacional, La Paz, Baja California Sur, México.

Cubillos, L. A, Aguayo, M., Neira, M., Sanhueza, S., \& Castillo-Jordán, C. (2009). Verificación de la edad y crecimiento del besugo Epigonus crassicaudus (de Buen, 1959) admitiendo error en la determinación de la edad. Revista de Biología Marina y Oceanografia, 44(2), 417-427.

Charnov, E. L., \& Gillooly, J. F. (2004). Size and temperature in the evolution of fish life histories. Integrative and Comparative Biology, 44, 494-497.

Csirke, J. (1980). Introducción a la dinámica de poblaciones de peces (Documento Técnico de Pesca 192). Roma, Italia: FAO.

de León, M. E. (2005). Variabilidad temporal de los parámetros poblacionales de la langosta espinosa del Caribe Panulirus argus (Latreille, 1804) en aguas de Cuba (Tesis de Doctorado). Centro de Investigaciones Biológicas del Noroeste, Instituto Politécnico Nacional, La Paz, Baja California Sur, México.

do Santos, A., Pessanha, A., da Costa, M., \& Araújo, F. (2004). Relação peso-comprimento de Orthopristis ruber (Cuvier, 1830) (Teleostei, Haemulidae) na Baia de Sepetiba, Rio de Janeiro, Brasil. Revista Brasilera de Zoología, 21(2), 185-187.

Ehrhardt, N. (1981). Métodos de análisis de las estadísticas de captura y esfuerzo de pesca y su aplicación en modelos globales de pesquerías (Curso sobre Biología Pesquera). La Paz, Baja California Sur, México: Centro Interdisciplinario de Ciencias Marinas, Instituto Politécnico Nacional.

Gayanilo, F. C. Jr., Sparre, P., \& Pauly, D. (1996). FAOICLARM stock assessment tools (FiSAT). User's guide. FAO Computerized Information Series (Fisheries) 8. Rome, Italy: FAO.

Gómez, G., Guzmán, R., \& Chacón, R. (2002). Algunos aspectos de la biología reproductiva y poblacional del torroto, Genyatremus luteus (Bloch, 1797) (Pisces: Haemulidae) en el golfo de Paria, Venezuela. Zootecnia Tropical, 20(2), 223-234.

González, L. W., Eslava, N., Guevara, F., Díaz, F., \& Rodríguez, J. M. (2017). Evaluación de la pesquería artesanal de El Tirano, isla de Margarita, Venezuela. Boletín del Centro de Investigaciones Biológicas (En prensa).

González, L. W., Eslava, N., \& Guevara, F. (2001). La pesca artesanal del pulpo (Octopus spp.) en El Tirano, Isla de Margarita, Venezuela. Ciencia, 9(1), 18-27.

Gulland, J., \& Holt, S. (1959). Estimation of growth parameters for data at unequal time intervals. Journal $d u$ Conseil International pour l'Exploration de la Mer, 25, 47-49.

Gulland, J. (1971a). Manual de métodos para la evaluación de las poblaciones de peces. Zaragoza, España: FAO.

Gulland, J. (1971b). The fish resources of the oceans. Surrey, England: Fishing News Books Ltd.

Kaufmann, K. W. (1981). Fitting and using growth curves. Oecología, 49, 293-299.

Lieske, E., \& Myers, R. (1994). Collins Pocket Guide. Coral reef fishes. Indo-Pacific \& Caribbean including the Red Sea. New Jersey, USA: Haper Collins Publisher.

Lindeman, K. (1989). Coastal construction, larval settlement, and early juvenile habitat use in grunts, snappers and other coastal fishes of southeast Florida. Bulletin Marine Science, 44(2), 1068-1089.

Maroñas, M. (2006). Crecimiento individual en peces. Cátedras de Ecopoblaciones. Recuperado de http:// www.fcnym.unlp.edu.ar/catedras/ecopoblaciones. 
Musick, J. A. (1999). Criteria to define extinction risk in marine fishes. Fisheries, 24(12), 6-14.

Pauly, D. (1979). Theory and management of tropical multispecies stocks: a review, with emphasis on the Southeast Asian demersal fisheries. International Center for Living Aquatic Resources Management, Studies and Review, 1, 1-35.

Pauly, D. (1980). On the interrelationships between natural mortality, growth parameters and mean environmental temperature in 175 fish stocks. International Council for the Exploration of the Sea, 39(2), 175-192.

Pauly D. (1983). Length-converted catch-curves. A powerful tool for fisheries research in the tropics (Part I). Fishbyte, 1(2), 9-13.

Pauly, D. (1993). Fishbyte section editorial. Naga, 16 $(2-3), 26$

Pauly, D., \& Munro, J. L. (1984). Once more on the comparison of growth in fish and invertebrates. Fishbyte, 2(1), 21.

Pinheiro, H. T., Gasparini, J. L., \& Joyeux, J. C. (2010). Reef fish mass mortality event in an isolated island off Brazil, with notes on recent similar event sat Ascension, St Helena and Maldives. Marine Biodiversity Records, 3, 1-4.

Pope, J. A., Margetts, A. R., Hamley, J. M., \& Akyuz, E. F. (1983). Manual de métodos para la evaluación de las poblaciones de peces. Parte 3. Selectividad del arte de pesca. (Documento Técnico de Pesca 41 Rev. 1). Roma, Italia: FAO.

Powell, D. (1979). Estimation of mortality and growth parameters for the length frequency of a catch. Rapport procès-V Réunion Conseil International pour L'Exploitation de la Mer, 175, 167-169.

Prince, P., Lee, D., \& Conser, R. (1987). Estimating age and growth rate of Atlantic blue marlin (Makaira nigricans): progress and future work plan. Collective Volume of Scientific Papers ICCAT, 26(2), 426-435.

Ricker, W. (1975). Computation and interpretation of biological statistic of fish population. Department of Environmental Fisheries and Marine Service. Bulletin of Fisheries Research Board of Canada, 191, 382.
Slack-Smith, R. J. (2001). Fishing with traps and pats. (Training Series 26). Rome, Italy: FAO.

Sparre, P., \& Venema, S. (1997). Introducción a la evaluación de recursos pesqueros tropicales. Parte 1. Manual (Documento Técnico de Pesca 306. 1 Rev. 2). Roma, Italia: FAO.

Taylor, C. (1958). Cod growth and temperature. Journal du Conseil International pour l'Exploration de la Mer, 23, 366-370.

Tordecilla-Petro, G., Sánchez-Banda, S., \&. Olaya-Nieto, Ch. (2005). Crecimiento y mortalidad del moncholo (Hoplias malabaricus), en la Ciénaga Grande de Lorica, Colombia. Revista MVZ Córdoba, $10(2), 623-632$.

Tresierra, A., \& Culquichicón, Z. (1993). Biología Pesquera. Trujillo, Perú: Editorial Libertad.

von Bertalanffy, L. (1938). A quantitative theory of organic growth. Human Biology, 10(2), 181-213.

Villa, M. (1967). Aspectos geográficos de las Dependencias Federales. Caracas: Corporación Venezolana de Fomento.

Walpole, R., Myers, R., Myers, S., \& Ye, K. (2007). Probability \& Statistics for Engineer \& Scientists. New Jersey, USA: Pearson Prentice Hall.

Wetherall, J. (1986). A new method for estimating growth and mortality parameters from length-frequency data. Fishbyte, 4, 12-14.

Zar, J. (2009). Biostatistical analysis. New Jersey, USA: Prentice Hall

Zavatteri, A. (2010). Estudio de edad y crecimiento de la raya hocicuda Dipturus chilensis (Guichenot, 1848) en el Atlántico Sudoccidental $\left(34^{\circ}-55^{\circ} \mathrm{LS}\right.$ y $52^{\circ}-69^{\circ}$ $L W)$ (Tesis de Pregrado). Universidad Nacional de Mar del Plata, Argentina.

Zúñiga, M. (2009). Dinámica poblacional del Dorado (Coryphaena hippurus) en Baja California Sur, México: implicaciones para su manejo (Tesis de Doctorado). Centro Interdisciplinario de Ciencias Marinas, Instituto Politécnico Nacional, La Paz, Baja California Sur, México. 